\title{
Nonlinear dynamics of self-centring rocking steel frames using finite element models
}

\author{
L.T. Kibriya ${ }^{\mathrm{a}}$, C. Málaga-Chuquitaype ${ }^{\mathrm{a}, *}$, M.M. Kashani $^{\mathrm{b}}$, N.A. Alexander ${ }^{\mathrm{c}}$ \\ ${ }^{a}$ Department of Civil and Environmental Engineering, Imperial College London, London, United Kingdom SW7 $2 A Z$ \\ ${ }^{b}$ Faculty of Engineering and the Environment, University of Southampton, Southampton, United Kingdom SO17 1BJ \\ ${ }^{c}$ School of Civil, Aerospace and Mechanical Engineering, University of Bristol, Bristol, United Kingdom BS8 1TR
}

\begin{abstract}
Rocking post-tensioned steel frames capitalise on the use of rocking joints, and unbonded post-tensioning strands to provide self-centring action. Investigations on the complex and unconventional nonlinear dynamics of tied rocking steel frames, exclusive of supplemental damping methods, are presently limited. Increasing levels of energy-dissipation reduce the probability of observing nonlinear dynamic phenomena such as co-existing (high/low) amplitude responses at and around the system's nonlinear resonance. To this end, a finite element (FE) modelling framework is presented, validated and extended to multi-storey steel buildings. It is shown that the simulation strategies proposed enable an accurate representation of the complex nonlinear dynamics of self-centring structures, over a wide range of excitation frequencies and amplitudes. The methodology, applied to multi-storey steel frames, captures the presence of sub-harmonic resonances and higher-modes. It is also demonstrated that the additional demands observed in the rocking columns are the consequence of the asymmetry of the member boundary conditions.
\end{abstract}

Keywords: finite-element; self-centring; rocking; nonlinear dynamics; post-tensioned moment frame

\section{Introduction}

The staggering social and economic impacts of earthquakes have recently inspired a growing interest in smart structural systems [1]. Rocking structures, in particular, are earthquake resilient systems that utilise elastic gap-opening mechanisms at their connections to soften the structural response and enable a building to remain operational after a seismic event. Furthermore, rocking self-centring steel moment frames 5 employ rocking connections at the base of the columns and beam-to-column interfaces. This system utilises unbonded post-tensioned tendons to tie members together in order to develop the necessary moment resistance, and provide the restoring force required to centre the structure back to its initial position (plumb), in the aftermath of the earthquake event. Energy dissipation is typically provided by supplemental means using replaceable components, rather than through the yielding (damage) of primary structural members. This means that the inelastic structural deformations under lateral forces can be reduced or even prevented [2].

Research encompassing various aspects of the design and behaviour of different classes of rocking structures has spanned over the last two decades. While some of this research did not consider the self-centring 15 effects of post-tensioning and focussed on free-standing rocking structures [3, 4, 5], other studies examined the response of a range of self-centring systems, including steel-braced, pre-cast concrete and timber frames, as well as concrete and timber shear wall configurations with significant levels of energy-dissipation coming from sacrificial components $[6,7,8,9,10,11]$. The presence of energy-dissipation diminishes the inherent

\footnotetext{
* Corresponding author

Email address: c.malaga@imperial.ac.uk (C. Málaga-Chuquitaype)
} 
nonlinearity of a rocking structure, and limits the examination of its raw nonlinear dynamic response. Furthermore, a considerable amount of research has studied the resilience of individual steel joint assemblies, following a concentrated plasticity and phenomenological approach, that does not lend itself to scalability of responses $[12,13,14]$. Consequently, a majority of the studies were concerned with predicting the response of rocking steel buildings to ground motion excitations through analytical approximations $[15,16,17,18]$. By contrast, this paper shows that frequency response functions (FRFs) generated using numerical modelling techniques are a viable and convenient approach that allows an insight into the mechanics of tied rocking frames.

Alexander et.al. [19] carried out a series of numerical, analytical, and experimental studies to examine the nonlinear dynamics of a single-storey rocking post-tensioned steel moment frame exclusive of any supplemental energy-dissipation [19, 20, 21]. A quarter-scale physical model comprising steel square hollow sections representing a single bay and storey from the PRESSS building [6, 22, 23] was adopted. Discrete frequency sine-sweep analyses were performed in order to confirm the rocking model's long-term high cycle performance. A low-order analytical model was also presented to estimate the dynamic response of the rocking frame as an equivalent single-degree-of-freedom (SDOF) tied rocking block [19]. Although this model was highly effective in the prediction of a SDOF system's response, its implementation can be mathematically and computationally complex, especially for multi-degree-of-freedom (MDOF) structures. Moreover, the analytical study was limited to a single-storey system and the response of multi-storey steel structures under pure rocking requires further examination.

Similarly, there have been few attempts at proposing modelling techniques to capture the highly nonlinear behaviour of rocking frames, utilising finite and discrete element frameworks [12, 15, 16, 21, 24, 25, 26]. However, many of these methodologies do not go into detail regarding the overall model assembly, the calculation of associated variables, and the selection of parameters for the analysis. Moreover, numerical procedures, in the context of nonlinear dynamic analysis of tied rocking steel structures, have not been 45 explicitly discussed in previous research. A successful simulation methodology should be able to capture the fundamental features of the nonlinearity in the system under strong ground motion. The multi-spring contact element approach is the most commonly implemented strategy in finite element simulations for the in-plane rocking motion of steel frames. The geometry of this element comprises of an evenly distributed series of springs, confined between rigid links at the end surface of the adjoining structural members. The 50 springs are defined typically to offer no resistance in tension and have an elastic response in compression. This modelling approach results in the contact zone being infinitely stiff, and the opening joints rotating around the pivot point at the member edges. Therefore, the motion simulated by these models conforms to the rocking principles introduced by Housner [27].

An alternative approach was presented in [24] for the numerical modelling of rocking connections, using the finite element programme, RUAUMOKO [28]. The numerical model was compared with static experimental results of beam-column connection sub-assemblies and a multi-storey reinforced concrete frame structure with draped tendons. The method was demonstrated to successfully represent the nonlinear joint behaviour of the connections, account for the neutral axis shift in the contact area, and capture the beam 60 elongation and post-tensioned tendon lengthening effects during ground motion excitation. Alternatively, the experimental and analytical models of the steel frame described previously [19, 20, 21] were simulated using the Discrete Element (DE) software UDEC [29]. Mass-proportional Rayleigh damping of 5\% was applied to the model. It was concluded that the DE model adequately replicated the qualitative aspects of the physical and analytical response. However, the input sensitivities concerning material properties and damping 65 models, observed in the discrete-element analysis represent an important handicap of this framework in the context of modelling nonlinear rocking steel frames. This limits the reliability of the modelling method and can lead to inaccurate response predictions. Therefore, it is critical to develop generalisable modelling considerations which can ultimately facilitate the practical application of post-tensioned rocking steel buildings.

This paper investigates the potential use of Finite Element modelling to predict the complete fundamen- 
tal nonlinear response of pure rocking steel frames with particular attention to nonlinear resonance curves (frequency response functions). It is noted that this method of analysis has not been formerly utilised in the study of post-tensioned rocking steel systems and this paper will address this shortcoming. First, numerical simulation strategies are compiled and validated against the experimental, analytical, and discrete-element 75 responses of a selected single-storey rocking steel frame. Next, the procedures are applied to multi-storey tied rocking moment frames. The resulting structural behaviour is compared with that of conventional rigid moment-resisting steel frames (MRFs). Additional effects in the rocking frame bending moments due to the interaction between structural members with the different end conditions (rocking column, continuous column, and rocking beam) are observed and investigated. The results presented demonstrate that the column

so bending moments are impacted by these beam-column interactions, whereas the storey displacements and shear forces are qualitatively similar to those observed in a conventional MRF. These effects have not been explored in preceding studies and are pertinent as they can inform the provision of practical steel design guidelines for post-tensioned rocking frames.

\section{Numerical Modelling Approach}

\subsection{Selected Single-storey Frame}

The numerical studies presented in this paper required a well-documented physical rocking steel momentresisting frame benchmark study. For this purpose, the model had to be extensively explored for both static and dynamic analyses. Structural simplicity was an important selection criteria, evidenced by structural regularity and the absence of complex supplementary energy-dissipation elements, devices or mechanisms. This would reduce modelling and analysis uncertainties, enabling a more fundamental understanding of the nonlinear dynamic phenomena. In consideration of these attributes, the physical, numerical, and analytical research conducted by Alexander et al. [19, 20, 21], was selected for this research. The aforementioned series of investigations were among the first to study in detail, the pure rocking response of a complete steel moment frame structural assembly. Therefore, this study provides feasible baseline results to validate numerical models.

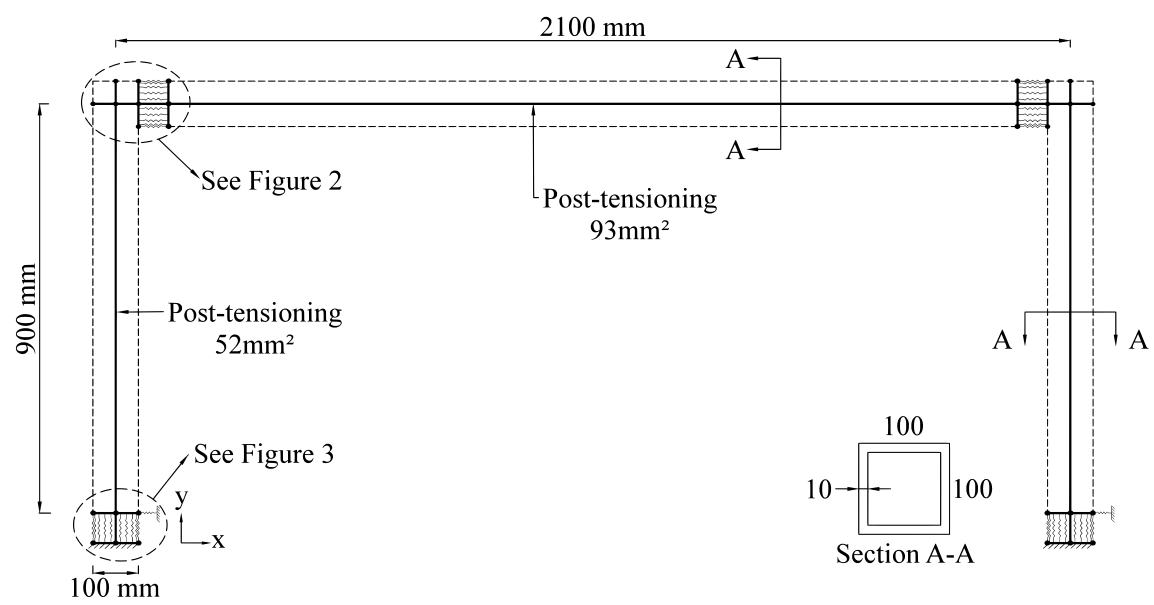

Figure 1: One storey, single bay model schematic with members and connection details.

The structural model employed as a benchmark is a one-storey quarter-scale single-bay planar steel frame, comprising of moment-resisting rocking connections at the beam-to-column and column-to-foundation interfaces. The height of the frame is $0.9 \mathrm{~m}$ and its bay width is $2.1 \mathrm{~m}$ (Figure 1). These values correspond to an aspect ratio of 0.4 . The detailed design of the original model building was carried out in accordance with design guidelines presented as part of the PRESSS research programme [23], and it represented a single 
Table 1: Spring Position and Weighting for a 2- and 10-spring gap element [24]

\begin{tabular}{ccccc}
\hline No. of Springs & \multicolumn{2}{c}{ Gauss Quadrature } & \multicolumn{2}{c}{ Lobatto Integration } \\
\hline \multirow{3}{*}{ 2 Springs } & Absciccas & Weights & Absciccas & Weights \\
\cline { 2 - 5 } 10 Springs & \pm 0.577 & 1.000 & \pm 1.000 & 1.000 \\
\hline & \pm 0.149 & 0.296 & \pm 0.165 & 0.328 \\
& \pm 0.433 & 0.269 & \pm 0.478 & 0.292 \\
& \pm 0.679 & 0.219 & \pm 0.739 & 0.225 \\
& \pm 0.865 & 0.150 & \pm 0.920 & 0.133 \\
& \pm 0.974 & 0.067 & \pm 1.000 & 0.022 \\
\hline
\end{tabular}

bay and storey from the PRESSS structure. The beam and column elements of the model frame were both made from hollow steel square sections, with dimensions of $100 \times 100 \times 10 \mathrm{~mm}^{3}$. A tendon pre-tension and cross section of $115 \mathrm{kN}$ and $93 \mathrm{~mm}^{2}$ for beam tendons, and $64 \mathrm{kN}$ and $52 \mathrm{~mm}^{2}$ for column tendons was employed. The applied mass on the frame was two tonnes. The specimen was tested at the University of Bristol Laboratory for Advanced Dynamics Engineering (BLADE) [19].

\subsection{Modelling Specifications}

The finite element programme OpenSees [30], was employed for the development of all numerical models presented herein. Planar frames with three-degrees-of-freedom per node were defined. The schematic for the numerical models of rocking connections are presented in Figures 2 and 3. The Lobatto and Gauss integration schemes introduced by Spieth et al. [24], were applied to distribute the members stiffness along the rocking surfaces. Preliminary studies demonstrated that either of the integration schemes can be selected the simulation of rocking surfaces. Varying number of springs were modelled ranging from 2 to 10 springs in order to assess the effects of different stiffness distributions (Table 1). The value for the number of gap elements along a rocking surface was calculated by dividing the length of the rocking surface by a factor of 10. It was found that the number obtained generally resulted in the formation of realistic compression zones at the rocking surfaces compared with experimental results and analytical approximations. All degrees of freedom were constrained at the foundation level to simulate a fixed base beneath the rocking interface. The end node at the base column joints were horizontally restrained to simulate a roller support in the Xdirection in order to prevent slip between adjacent rigid links. Different types of end conditions were tested in order to study their effect on the global behaviour of the frames. It was determined that the resulting static and dynamic responses were not sensitive to this detail. Vertical displacement restraints were added for the zero-length elements between the beam and column to facilitate shear transfer. This was achieved by using the equal degree-of-freedom constraint (master-slave nodes) for adjacent nodes between the beam-end rigid links representing the gap opening. Nodal masses were defined in the horizontal and vertical degrees of freedom, and lumped at the top of each column element. Corotational geometric transformation was applied for columns and beams to account for geometric nonlinearities, while linear transformation was used for the rigid links [30].

The columns and tendons were modelled as continuous elements along the frame heights. The posttensioning tendons were modelled using Corotational Truss Elements with an initial stress uniaxial material (Steel02 material in OpenSees [30]), and were anchored at points rigidly offset half the column and beam depths outside the frame centreline (Figures 1 and 2). An alternative model was developed for posttensioning anchored at the frame centreline in order to study the effects of the offsets (Figure 3). Structural elements were modelled with their respective material elastic moduli and yield strengths. S275 steel with an elastic modulus of $210 \mathrm{GPa}$ was used for the beam and column members [21]. Elastic beam-column elements were used for modelling the beams, columns, and rigid links. Elastic Perfectly Plastic material was defined for the gap element, with no stiffness in tension. Alternative models of gap elements included the Elastic No-Tension material, and hysteretic material. It was observed that material selection for the gap elements 


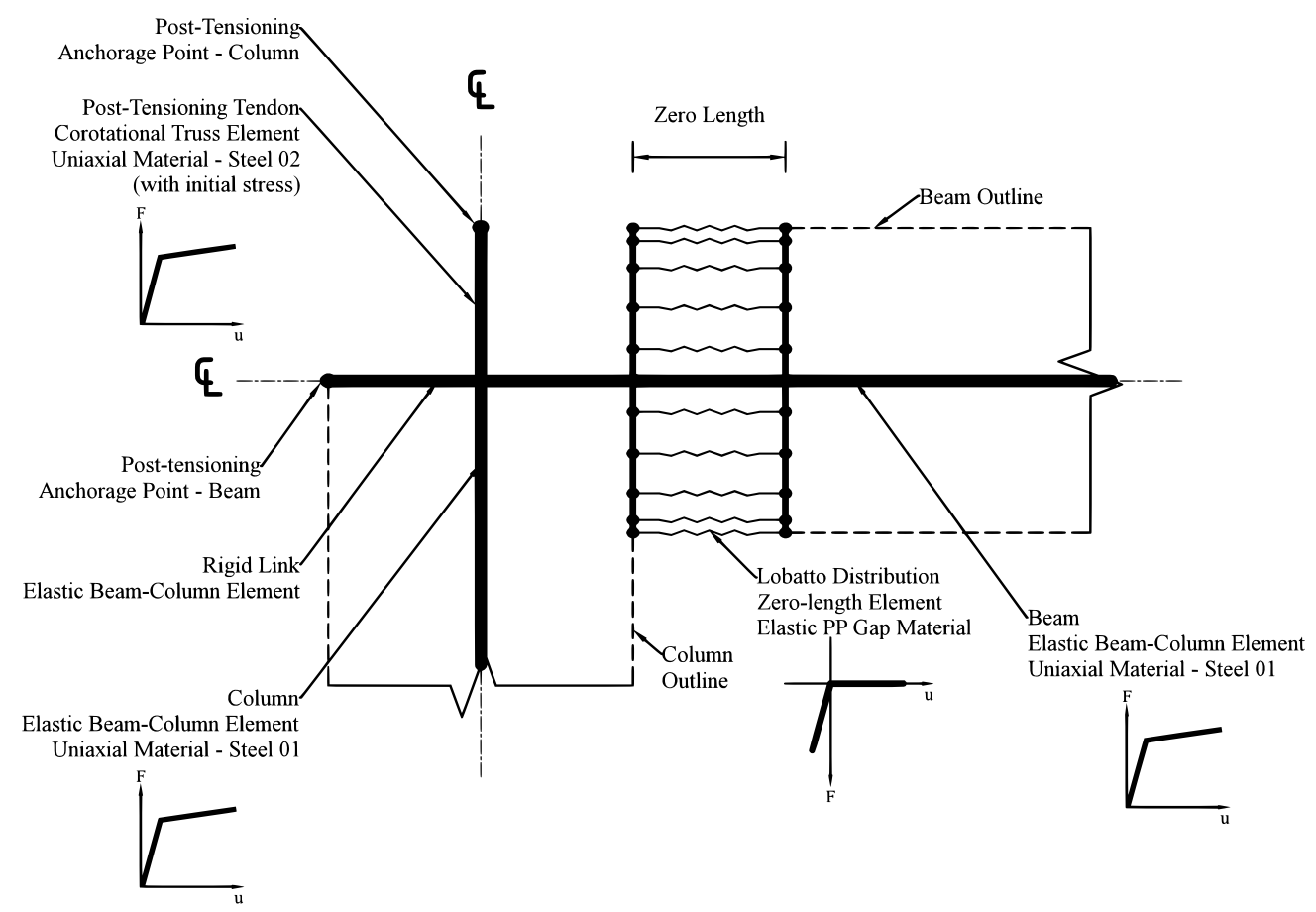

Figure 2: Beam-column connection detail with Post-Tensioning (PT) rigidly offset from centreline.

did not have a major impact on the dynamic results. The stiffness properties of each spring element were calculated using Equations 1 and 2 below.

$$
\begin{gathered}
K_{\text {spring }, i}=\frac{E A}{2 L_{I}} \cdot w_{i} \\
F s_{\text {spring }, i}=\frac{F_{y} A}{2} \cdot w_{i}
\end{gathered}
$$

Where, $E, A$ and $F_{y}$ are the Modulus of Elasticity, Cross-Sectional Area, and Yield Strength of the connecting member, respectively; $w_{i}$ is the weight of each spring assigned in accordance with Lobatto and Gauss integration schemes listed in Table 1 ; and $L_{I}$ is the influence length of the member which contributes to the stiffness of the corresponding rocking zone. Approximately one-third of the storey height for the columns and one-sixth of the bay widths for the beams, were applied. These values corresponded to approximately four times the column and two times the beam section depths. All proposed factors were obtained through sensitivity studies and comparisons of the numerical single and multi-storey models with experimental results and analytical approximations.

\subsection{Analysis Parameters}

The Static and Dynamic analyses include the definition and application of gravity loads, static lateral loads, and dynamic loads. Beam gravity loads were applied as nodal loads on the columns in order to simplify the model and reduce the processing time. Additionally, distributed beam loads were investigated and observed to not have an impact on the static and nonlinear dynamic results. The static lateral loads during pushover analysis were also applied at the nodes assuming linear first-mode distribution. Using the Equivalent Lateral Force procedure, the Base Shear was defined to be one unit in magnitude, distributed 


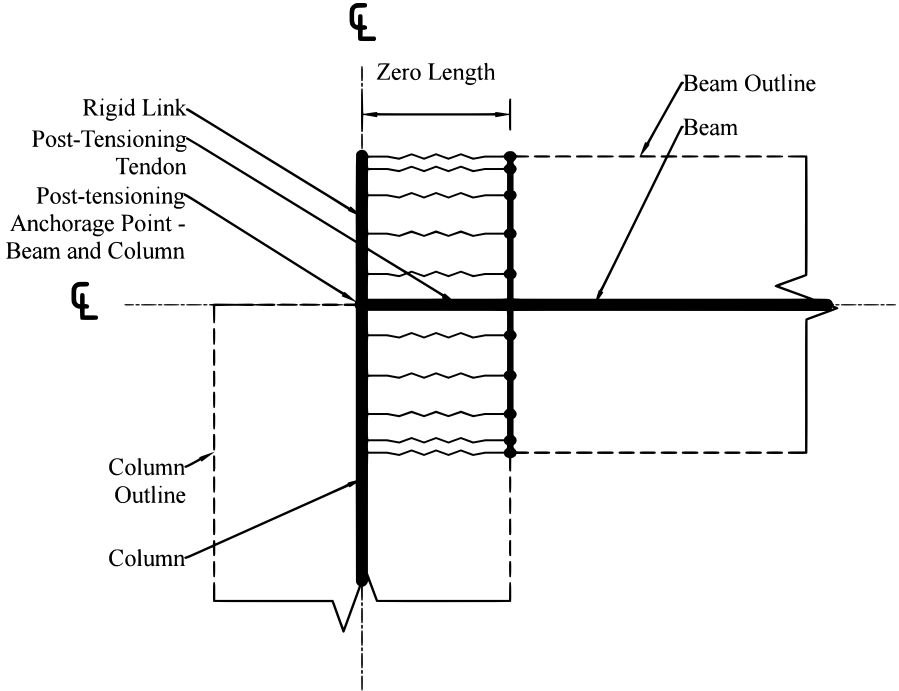

(a)

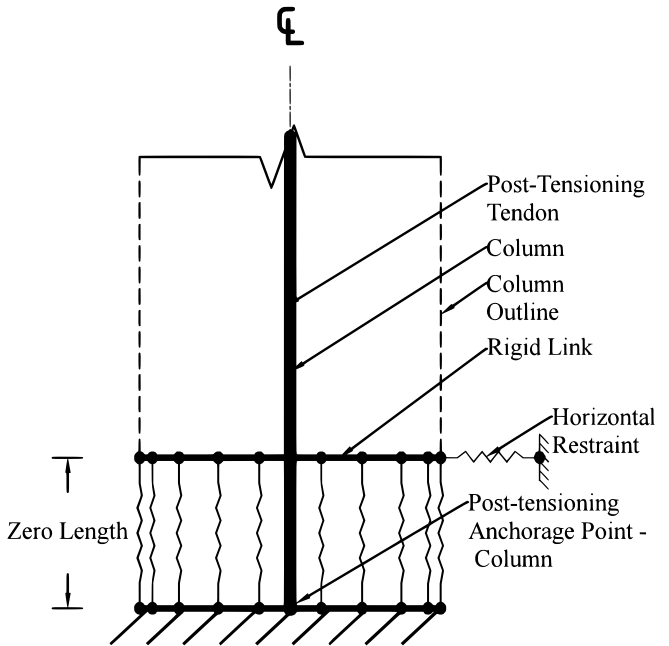

(b)

Figure 3: Beam-column connection without PT anchorage offset (a); Column-foundation connection detail (b).

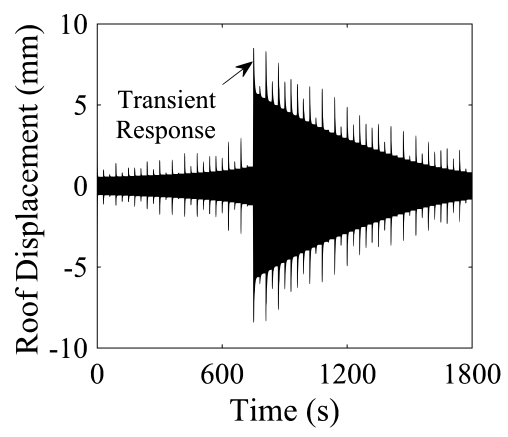

(a) Complete displacement responsehistory (transient and steady-state).

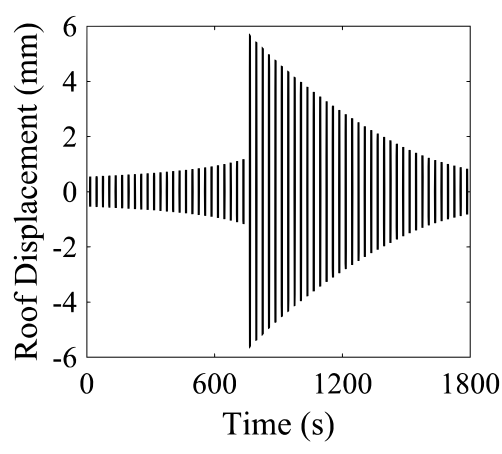

(b) Isolated steady-state responses

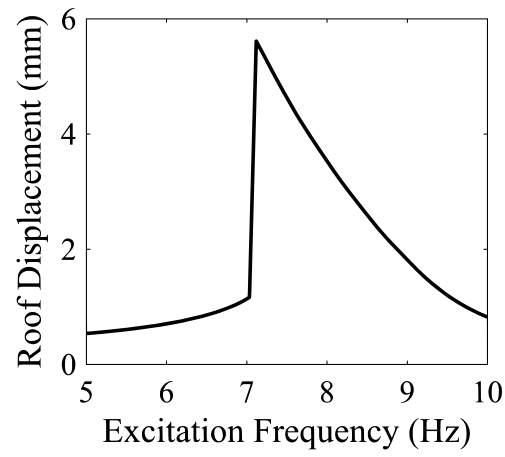

(c) Conversion of time to the corresponding frequency domain.

Figure 4: Generation of resonance response curves for a dynamic amplitude of $0.10 \mathrm{~g}$ for a single-storey rocking frame.

flo along the height of the frames based on the storey weights and heights above ground. A displacement control strategy was used to perform the nonlinear static analysis. A constant stiffness proportional damping of $5 \%$ was specified for all elements in the models.

Frequency Response Functions (FRFs) for structural engineering applications are typically generated using a sine-sweep or, more accurately, a sine-dwell input ground motion. Sine-dwell analysis is the discrete version of a sine-sweep analysis, where the frequency is not varied continuously but is incremented by discrete amounts, giving the structure time to reach a steady-state response. MATLAB [31] was used to generate input harmonic base motions. The automated algorithms developed allowed for the speedy definition of parameters including frequency range, time increment, sampling rate, duration and scaling.

The process of converting the response-history to resonance curves is illustrated in Figure 4 . The response-history obtained from the discrete sine-sweep analyses comprised of a transient and a steadystate dynamic response. The steady-state response was isolated to a user-defined time window, and the median response value corresponding to each increment of the forcing frequency was calculated. Finally, the 
time was converted to the frequency domain, and the forcing-frequency versus response quantity plots were generated. In the case of multi-storey frames, the maximum steady-state values of all structural response quantities (shears, moments, accelerations,and displacements) were plotted for each corresponding storey.

\section{Model Validation}

\subsection{Static Analysis and Monotonic Response}

The results of the static analysis for the single-storey model, described above, are illustrated in Figure 5. The roof displacements were plotted in terms of base shear against drift. The base shear (V) was normalised using the total weight of the frame (W). Influence lengths of $250 \mathrm{~mm}$ and $350 \mathrm{~mm}$ were used for beams and columns, respectively to calculate individual spring stiffness (Equations 1 and 2). The influence lengths were selected and calibrated based on the results of the experimental study. Sensitivity analyses were carried out for structural parameters such as the number of gap elements, influence length, post-tensioning anchorage offsets, and pretension force, discussed in further detail below. Through comparisons with the experimental study, it was demonstrated that the applied numerical modelling approach enabled an accurate representation of the pushover curve for a rocking post-tensioned steel moment frame.

It was observed that altering the initial pretension force of the columns did not have a significant impact on the static nonlinear response, whereas increasing the initial pretension force of the beam by a factor of 1.4 resulted in a $25 \%$ increase in the ultimate load. It was also noted that the anchorage point offsets did not have a significant influence on the pushover response of the structures as can be noted from Figure $5 \mathrm{c}$. The effects of the variation of integration schemes and stiffness distributions (number of gap-elements) were also analysed. It was observed that both the Lobatto and Gauss integration schemes resulted in similar responses when an appropriate number of spring elements were employed as demonstrated in Figure 5a. In order to determine the optimum number of zero-length elements, a number of spring elements ranging from 2 to 10 were defined for each model. Figure $5 \mathrm{~b}$ illustrates that a higher discretisation of the member stiffness distribution (more springs) leads to smoother pushover curves, and is accompanied by a decrease in the strength of the frame. It should be noted that, when using 2 springs with a Lobatto integration scheme, the connection rocks around the pivot points at 1, and no compression zones form. Hence, the inner lever arm at the rocking section is larger leading to higher forces at the opening for the same rotation at the joint (higher shear force). This explains why a higher shear resistance is observed for lower number of springs. The same phenomenon has been observed before by [24]. Importantly, a two-point contact configuration should be avoided as it does not distribute the stiffness accurately along the surface taking into account the compression zones which are formed during rocking. However these effects, approach an asymptote at around 6 springs, where the pushover curves begin to coincide. Beyond this point, distributing the member stiffness further along the surface does not yield any change in the structural response. Hence, the recommendation in Section 2 was presented regarding the need to divide the rocking surface by a factor of 10 to obtain a suitable baseline value for the number of gap elements. The selected number can be further calibrated based on the smoothness of the initial static results obtained.

\subsection{Dynamic Analysis and Frequency Response Functions (FRFs)}

A series of dynamic analyses were performed for low, medium, and high forcing amplitudes. The FRFs were developed using the methods described in Section 2, and are presented in Figure 6 in conjunction with the experimental results, discrete-element predictions, and analytical estimations of other studies [19, 20, 21]. The frequencies were normalised against the natural frequencies of the finite element models. The loweramplitude branch for the preceding experimental, analytical, and discrete-element models, was obtained using an increasing frequency, and the upper branch using a decreasing frequency sine-sweep ground-motion. It is important to note that the drop observed from the lower to higher branch in sine-sweep analysis is indeterminate in reality. This is because the solution in this region is unstable and cannot be obtained through numerical (finite element) simulations. Hence, the responses of nonlinear systems typically comprise of a 


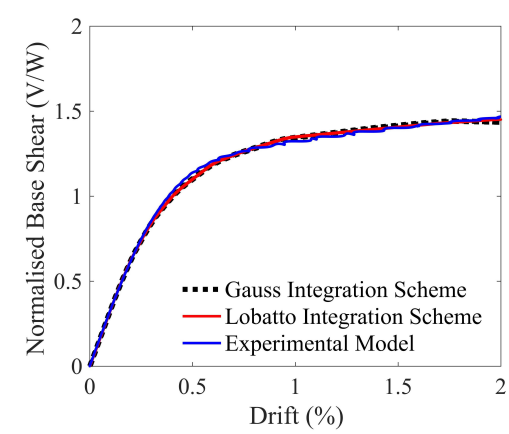

(a) Using Lobatto and Gauss integration schemes.

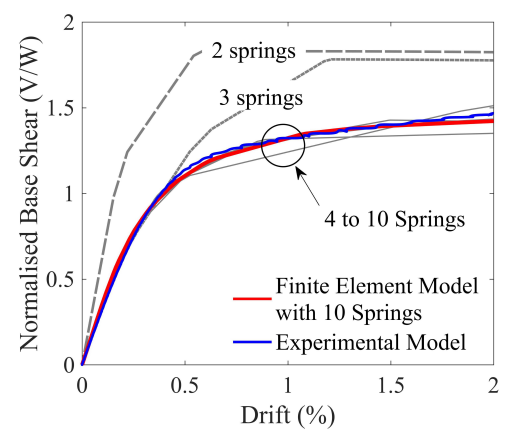

(b) Using varying number of zero-length gap-elements.

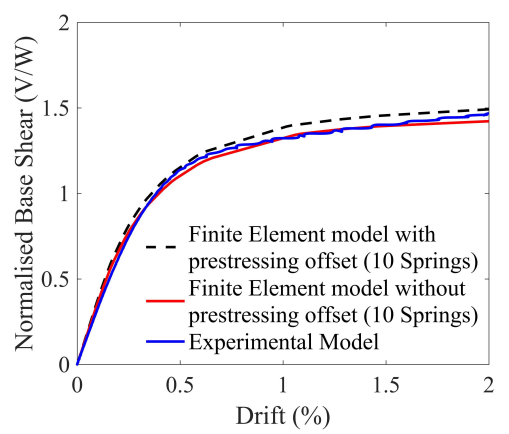

(c) Comparing responses with and without Post-Tensioning Offset Conditions.

Figure 5: Pushover curves for the single-storey steel rocking frames.

distinct upper and lower branch. These responses were obtained by introducing a high amplitude excitation for a finite duration, prior to the application of the desired frequency sweep loading in the proposed Finite Element models. Typically, an impulse load with an amplitude of $0.8 \mathrm{~g}$ to $1.2 \mathrm{~g}$ was applied for a duration of 5 to 7 seconds with a frequency around the resonant frequency of the particular response. An alternative procedure is to follow a similar method, but record individual responses for each frequency rather than applying a sweeping motion, following the impulse load. This would capture the secondary solution points individually in the increasing sine-sweep response range. The purpose of incorporating the impulse loading is to alter the initial conditions of the nonlinear frame (e.g. velocity) at the time the sine-sweep motion is applied, resulting in the overlapping areas in the dynamic structural response (upper resonance branch).

Figure $6 \mathrm{a}, \mathrm{b}$, and c illustrate the FRFs for the single-storey experimental, analytical and numerical (finite and discrete element) models for low, medium, and high forcing amplitudes. It can be observed that the Finite Element model captures the features of the physical and analytical models very well, with good agreement between the responses. It can also be noted from the figures that the response of the discrete-element model is divergent from the physical and analytical models, particularly for low amplitude forcing, where it results in considerably higher response amplitudes. Moreover, the experimental resonance curves do not match the analytical and numerical responses, at certain amplitudes. These discrepancies should be understood in the context of the difficulties experienced during experimentation to control the test specimen around resonance. Sensitivity analyses of the numerical test models also revealed that the initial pretension force does not have an effect on the dynamic characteristics of the models. This was also observed in previous studies [19].

By plotting the FRFs for a range of forcing amplitudes (Figure $6 \mathrm{~d}$ ), a backbone curve was constructed. A feature of backbone curves for nonlinear systems is that the lower amplitude responses approach the fundamental frequency of the system. It was noted that the fundamental frequencies of the finite element model and the analytical representation were approximately $8.4 \mathrm{~Hz}$. These were both close estimations for the physical frame's natural frequency of around $9 \mathrm{~Hz}$. The discrete-element model, on the contrary, resulted in a relatively higher natural frequency of around $11 \mathrm{~Hz}$. The striking similarities between the analytical and FE predictions validate the described numerical strategies. Thereafter, a series of dynamic analyses were performed with sinusoidal loading of low, medium and high amplitudes at the resonant frequency. The results were compared with the experimental pushover curves (Figure 7). The hysteresis curves of the rocking system demonstrate that the structure under consideration does not possess any inherent energy-dissipation (damping) capability. 


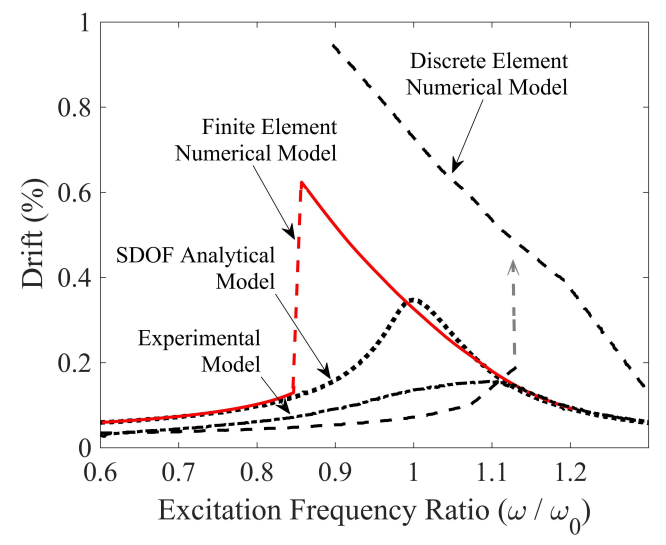

(a) Low-0.10 g forcing amplitude.

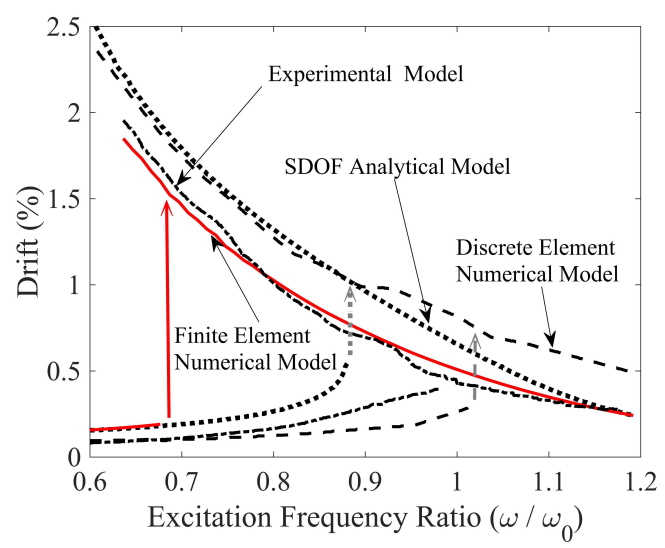

(c) High-0.26 g forcing amplitude.

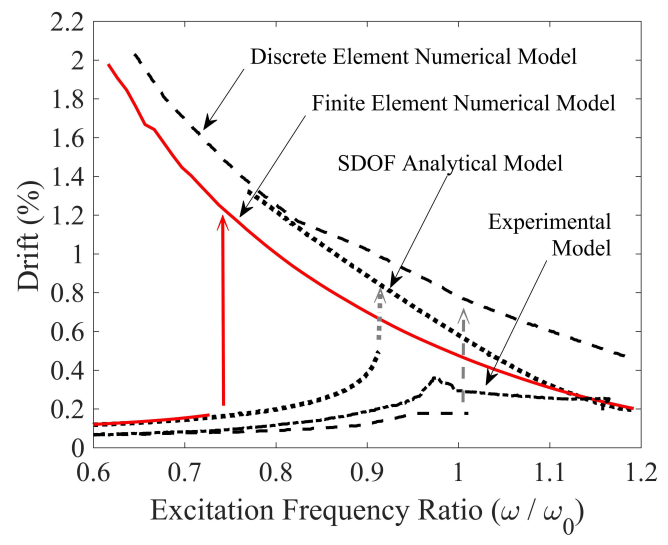

(b) Medium-0.20 g forcing amplitude.

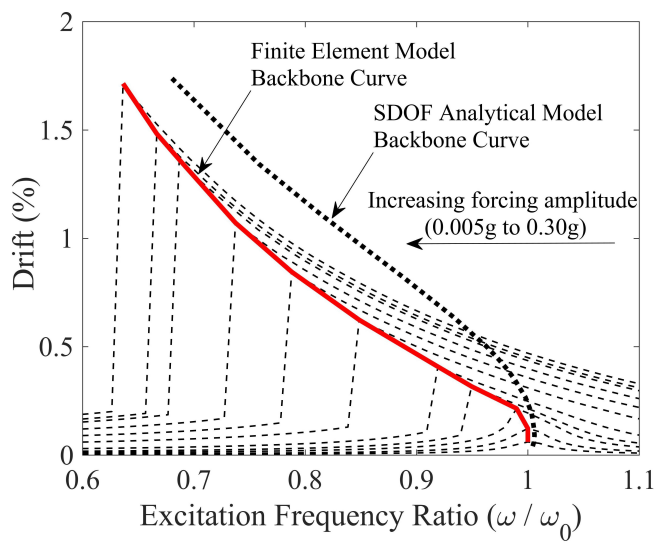

(d) Comparison of numerical and analytical backbone curves of the 1-storey rocking frame.

Figure 6: Frequency Response Functions (FRFs) for the 1-storey physical, numerical, and analytical models.

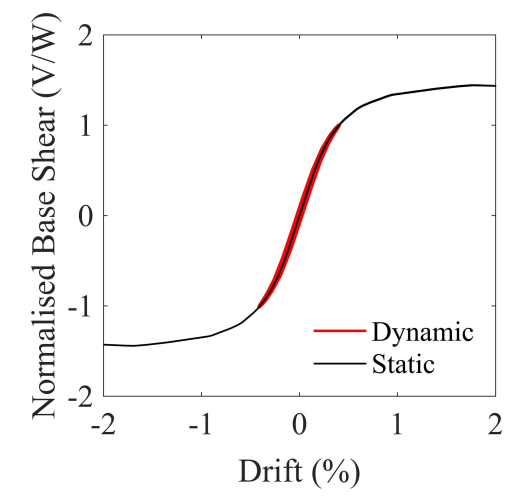

(a) Low-0.10 g forcing amplitude.

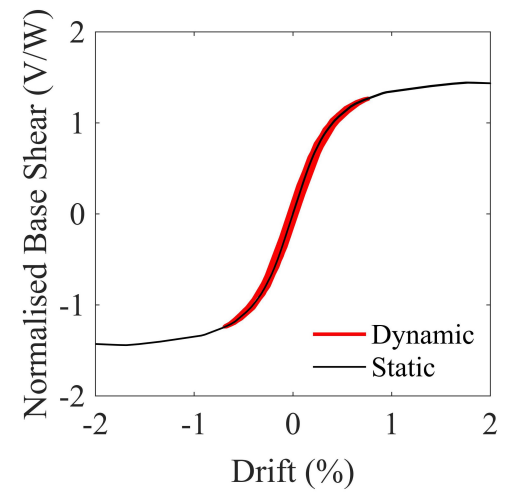

(b) Medium-0.20 g forcing amplitude.

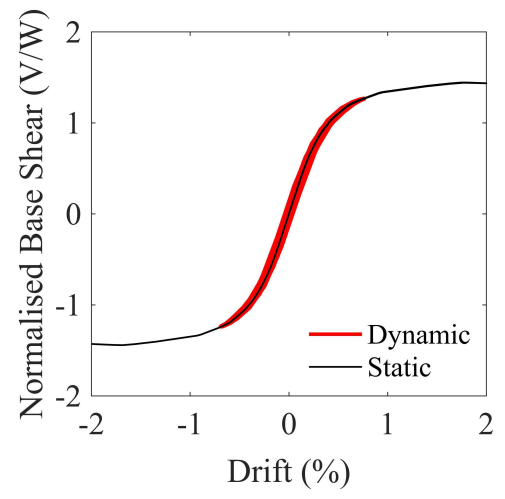

(c) High-0.30 g forcing amplitude.

Figure 7: Hysteresis curves for 1-storey rocking frame in response to cyclic (sinusoidal) loading. 


\section{Extension to Multi-storey Structures}

\subsection{Proposed Multi-storey Models}

The second stage of the research considered three, six and nine-storey single-bay planar steel frames, with moment-resisting rocking mechanisms at the beam-column and column-foundation connections. The aspect ratios and elevations for each of the structural models are illustrated in (Figure 8). Hollow steel square sections were used for the frame members, with identical sections for beams and columns. The inertial mass applied on the frames was two tons per bay. The higher storey models were designed based on the chosen single-storey benchmark frame, with a bay width of $1.2 \mathrm{~m}$ and storey height of $0.9 \mathrm{~m}$. Equivalent scale and ratios were used for all structures to maintain consistency. The sections used were $140 \times 140 \times 12 \mathrm{~mm}^{3}, 200$ x $200 \times 12 \mathrm{~mm}^{3}$, and $250 \times 250 \times 12 \mathrm{~mm}^{3}$ for the 3,6 and 9 storey frames. All members were designed to remain elastic for the duration of the highest forcing amplitude.

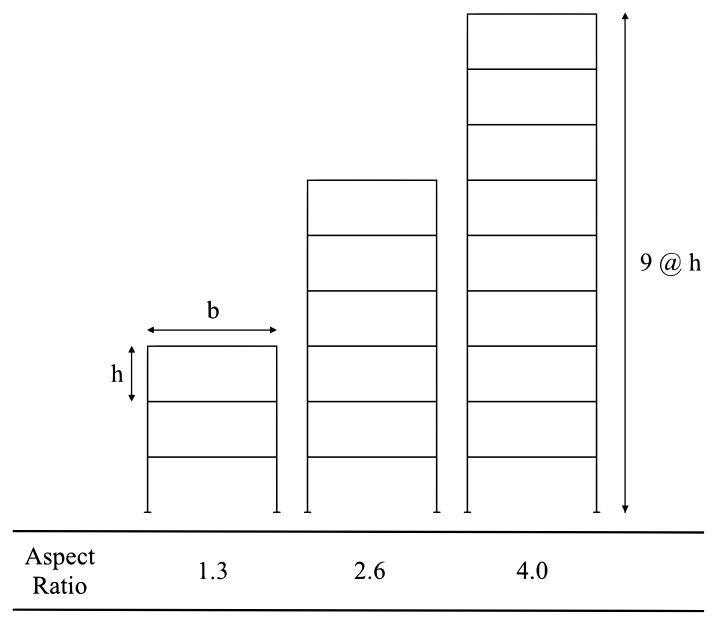

Figure 8: Elevations and aspect ratios for the 1, 3, 6, and 9-storey numerical models.

\subsection{Static Analysis and Monotonic Response}

The normalised base shear versus roof drift relationships for the multi-storey models studied herein, are

illustrated in Figure 9. The base shear was normalised against the weight of the buildings. Sensitivity analyses for parameters such as the influence length, member sizes, pretension force, and area of posttensioning tendons, were carried out for all the models, and selected results are presented in Figure 9. It was observed that altering the initial pretension force of the columns did not have a significant impact on the static response, whereas increasing the initial pretension force of the beam by a factor of 1.4 resulted in a $25 \%$ increase in the lateral strength of the frames. Additionally, doubling the cross-sectional area of the post-tensioning tendons in the columns, resulted in a response with negative stiffness at larger drifts. This leads to the counter-intuitive conclusion that a larger size of the post-tensioning tendons does not ensure a structure with greater strength and stability. Similarly, an increase in the initial force and/or area of post-tensioning tendons can have a detrimental impact on the response of rocking frames. This effect has been previously observed for rocking frames [32]. The increase in prestressing leads to a higher strain energy stored in the system. This causes the frame to rock with higher angular velocity and accentuates overturning after impact. This effect has been observed to be more pronounced for negative stiffness frames. It is worth noting that following the design assumptions, all of the steel structural elements remained elastic during the static pushover analyses. 


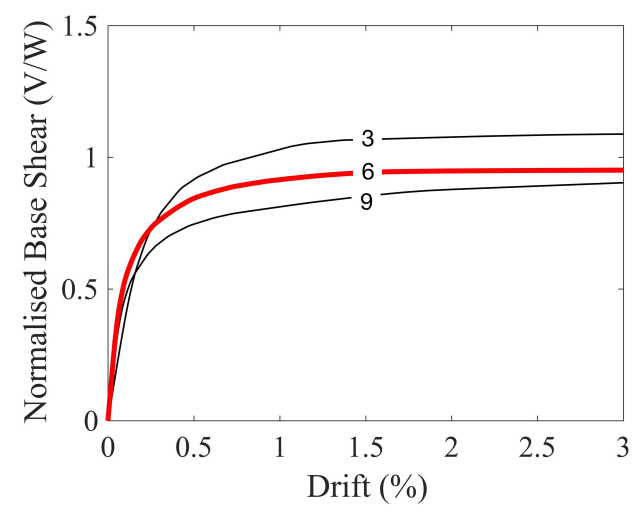

(a) 3-, 6-, and 9-storey buildings.

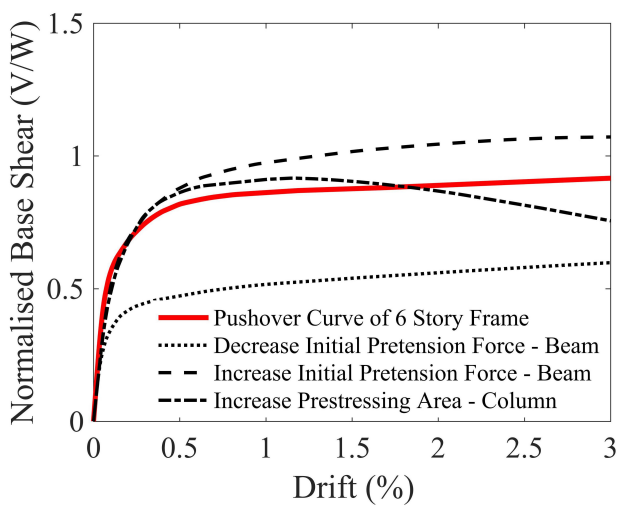

(b) 6-storey building with different pre-tension forces.

Figure 9: Normalised pushover response multi-storey numerical models.

\subsection{Dynamic Analysis and Frequency Response Functions (FRFs)}

A series of dynamic analyses were performed for low, medium, and high excitation amplitudes. The FRFs for the three, six, and nine-storey models are presented in Figures 10 and 11. The lower FRFs were obtained using the discrete sine-sweep analysis, and the upper branches using the decreasing frequency sine-sweep with initial impulse loading, as discussed previously. The FRFs were plotted in terms of roof and second-storey accelerations. It was observed that the displacement-based resonance curves were qualitatively similar to the single-storey frame responses. Moreover, the acceleration response plots for the threeand six-storey steel structures (Figures $10 \mathrm{a}, \mathrm{b}$ and $11 \mathrm{a}, \mathrm{b}$ ) demonstrate the presence of a single high-level peak at the respective resonant frequencies. By contrast, coexisting peaks can be recognised in the acceleration response amplitudes at the second storey level of the 9-storey frame (Peaks A, B, and C in Figure 11c).

Points A, B, and C correspond to frequencies of $1.27,2.02$, and $2.49 \mathrm{~Hz}$, with B representing the resonant frequency of the excitation amplitude $(0.30 \mathrm{~g})$. Point A occurs at a lower frequency than the resonant frequency and is a sub-harmonic resonance of the system. This sub-harmonic resonance can also be observed in the six-storey frame, and it becomes more prominent in the nine-storey frequency response functions. This demonstrates the importance of sub-harmonic resonances in more slender frames at high amplitude

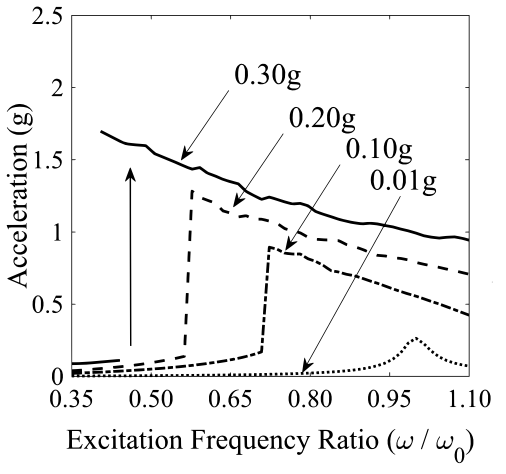

(a) 3-storey building.

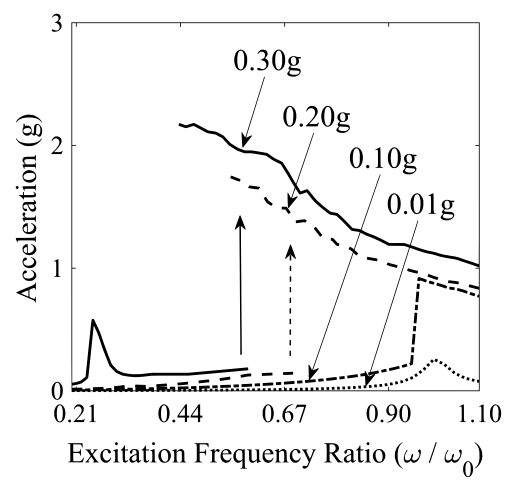

(b) 6-storey building.

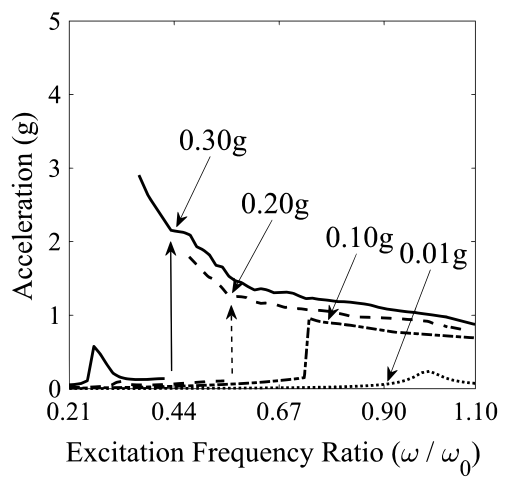

(c) 9-storey building.

Figure 10: Frequency response functions (FRFs) of multi-storey buildings illustrating roof accelerations for low- $0.10 \mathrm{~g}$, medium$0.20 \mathrm{~g}$, and high- $0.30 \mathrm{~g}$ amplitude excitations. 
excitation. It is important to note that selection of excitation frequency and time increments influences the secondary solutions recognised in the frequency sweep analyses. Frequency increments ranging from 0.01-0.04 Hz and time increments (dt) of 0.001 seconds, were applied for the models presented in this paper.

Point $\mathrm{C}$ was further evaluated by decreasing the time increment in the analysis. However, the peak (Point

C) was observed in these examinations as well. This phenomenon can be observed in nonlinear structures subjected to high amplitude excitations, and there is a high probability that it may be a product of the system's nonlinearity. Nonetheless, this conclusion is subject to further investigation.

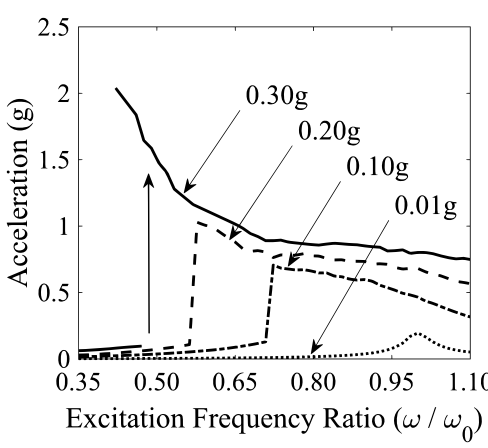

(a) 3-storey building.

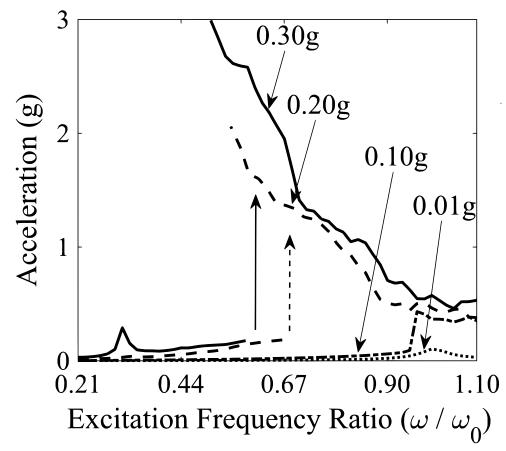

(b) 6-storey building.

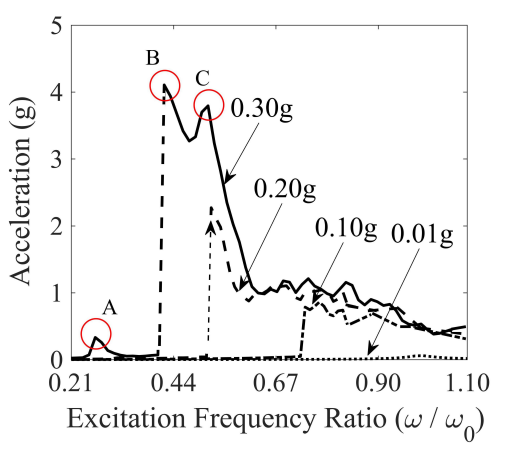

(c) 9-storey building.

Figure 11: Frequency response functions (FRFs) for multi-storey buildings illustrating maximum second-storey accelerations for low- $0.10 \mathrm{~g}$, medium- $0.20 \mathrm{~g}$, and high- $0.30 \mathrm{~g}$ amplitude excitations.

Moreover, the resonance response graphs were utilised to identify the fundamental frequencies of the building models using the maximum inflection points of low-amplitude FRFs. It is noted that the rocking joints do not open during the low-amplitude FRFs $(0.01 \mathrm{~g})$, and the frame behaves linearly. The natural frequencies of the three, six, and nine-storey frames were $5.8,5.2$, and $4.7 \mathrm{~Hz}$, respectively. The fundamental frequencies obtained from an eigenvalue analysis of the rocking frames were $6.1,5.3$, and $4.9 \mathrm{~Hz}$. The excitation frequency range of the rocking models was further expanded to study the influence of higher modes (Figure 12). Point D illustrates the second mode of the structures with frequencies of 20.6, 16.6, and 14.8 $\mathrm{Hz}$ for the three, six, and nine-storey buildings. Comparatively, second-mode frequencies of 22.8, 17.7, and $16.0 \mathrm{~Hz}$ were obtained from the eigenvalue analysis. Although the third mode cannot be clearly identified from the frequency response functions, the frequencies calculated from the eigenvalue analysis have been plotted (Point E) with values of 50.1, 37.0, and $31.5 \mathrm{~Hz}$. It is evident that the eigenvalue analysis provides reasonable estimates for the modal periods/frequencies of the nonlinear frame systems. It can be noted that the second mode occurs at around three times the first mode's frequency for all structural models. Furthermore, at higher modes, the frequency response functions are qualitatively similar to those of a linear structure with identical modal frequencies across all excitation amplitudes.

A linear-elastic model of a nine-storey fixed-base steel moment-resisting frame (MRF) with fully rigid joints was modelled with identical sections as those of the corresponding rocking frame. It is important to note that in reality, both structural systems would be designed differently to meet similar demands. The purpose of using consistent members and geometry for both structures was to highlight the qualitative differences in the fundamental behaviour of rocking frames and rigid MRFs. The FRFs of the rigid frame are illustrated in Figure 13. Contrary to the rocking structure, a fundamental frequency of $6.4 \mathrm{~Hz}$ for the linearelastic frame is constant across all forcing amplitudes. It is apparent that the rocking frame has greater flexibility than its steel MRF counterpart, which is expected because of the increased storey displacements due to the gap-opening mechanisms. The resonant frequencies identified for the rocking frames were further used to generate sinusoidal loading and perform harmonic analysis for the multi-storey numerical models. The comparison of the cyclic hysteretic responses for all multi-storey models with the respective static 


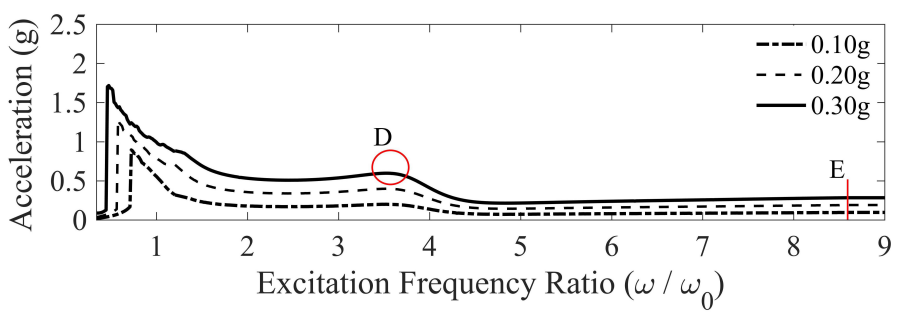

(a) 3-storey building.

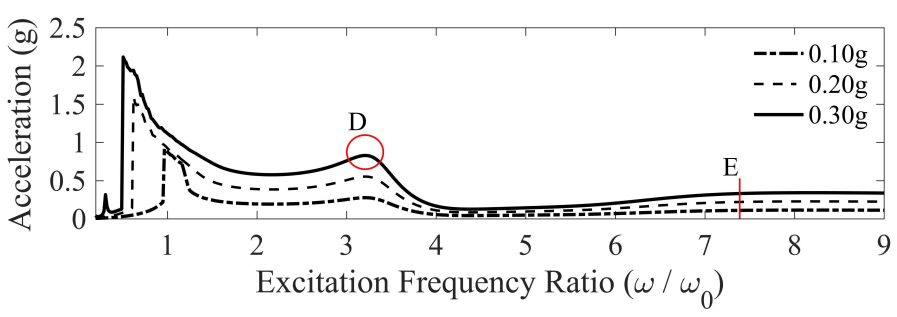

(b) 6-storey building.

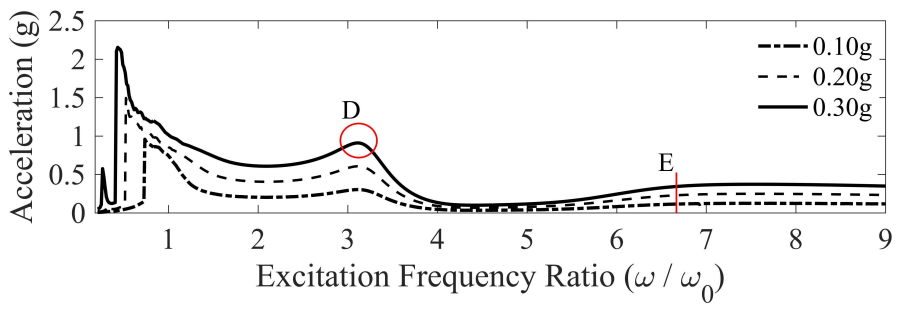

(c) 9-storey building.

Figure 12: Frequency response functions (FRFs) for multi-storey buildings illustrating roof accelerations for low- $0.10 \mathrm{~g}$, medium$0.20 \mathrm{~g}$, and high- $0.30 \mathrm{~g}$ amplitude excitations.

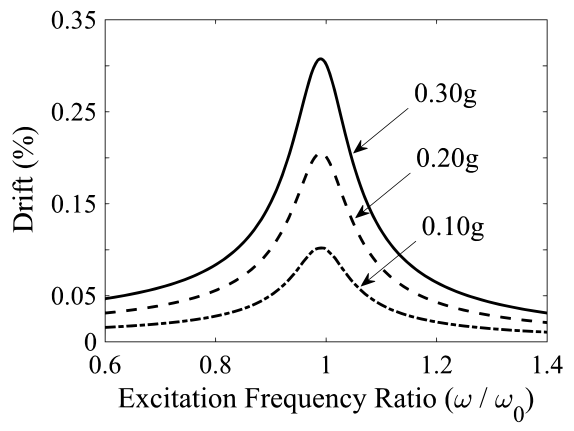

(a) Roof drifts.

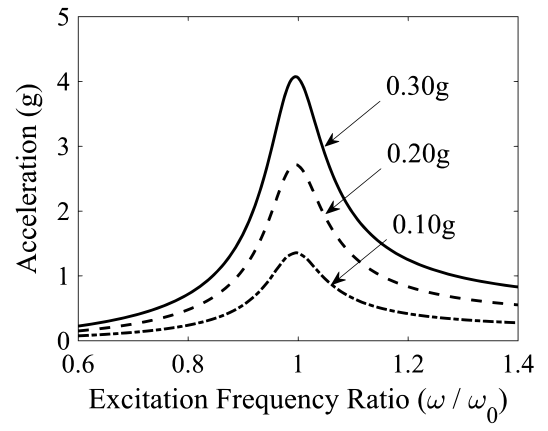

(b) Accelerations

Figure 13: Frequency response functions (FRFs) illustrating roof drifts and accelerations of a 9-storey fixed-base rigid frame for low- $0.10 \mathrm{~g}$, medium- $0.20 \mathrm{~g}$, and high- $0.30 \mathrm{~g}$ amplitude forcing. 
pushover were qualitatively similar to the single-storey behaviour. However, minor deviations in the cyclic response were observed at intersections of the linear and nonlinear branches of the pushover curve for the nine-storey model. These deviations were not as prominent in the three-storey steel structure and therefore can be attributed to sub-harmonic effects. Moreover, similar to the single-storey cyclic response (Figure 7), the graphs demonstrate that the structural systems do not possess significant energy-dissipation (damping) capability.

Maximum structural response quantities (e.g. Point B in Figure 11c) including displacements, drifts, accelerations, storey shears, and column bending moments for the three, six, and nine-storey steel frames are illustrated in Figures 14 to 16. Figure 17 presents the height-based responses of the nine-storey rigid frame, while response values for the nine-storey frame associated with Point $\mathrm{C}$ in Figure 11, are presented in Figure 18 for a high amplitude forcing. These graphs represent envelopes of the maximum steady-state magnitudes for each individual response quantity. It can be noticed that the displacements and shear forces or all frames are qualitatively similar to the responses observed in a conventional MRF (Figure 17). Also of note are the drift responses for the MRF which indicate more pronounced differences in the relative displacements between adjacent storeys (follow a more curved path). In comparison, the drift ratios for the rocking frames are relatively constant along the building heights. This is because the rigid connections lead to a stiffer structure where the behaviour of the beam and column members is dependent upon one another. In rocking moment frames, with openings between all member interfaces, the deformation of the structure follows a shape similar to that of a rigid rocking block, with the displacements increasing linearly with constant inter-storey drifts.
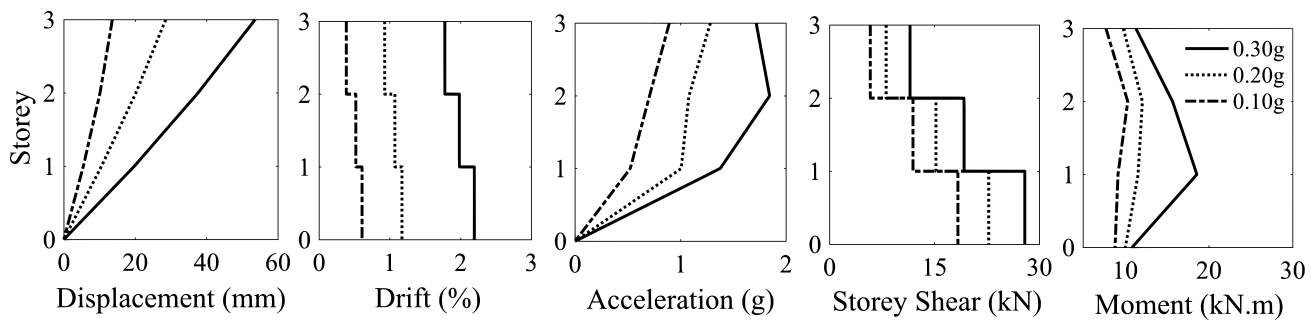

Figure 14: 3-storey structural response quantities for low- $0.10 \mathrm{~g}$, medium- $0.20 \mathrm{~g}$, and high- $0.30 \mathrm{~g}$ harmonic amplitude baseexcitation.
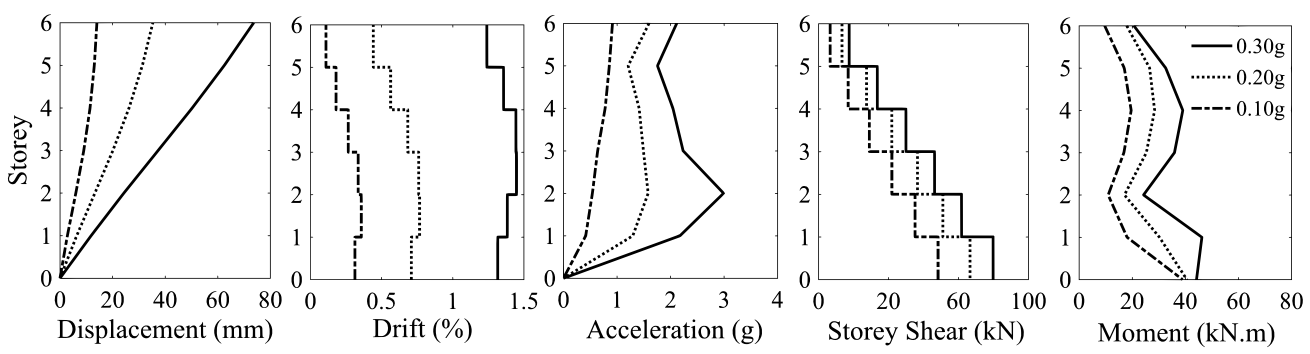

Figure 15: 6-storey structural response quantities for low- $0.10 \mathrm{~g}$, medium- $0.20 \mathrm{~g}$, and high- $0.30 \mathrm{~g}$ harmonic amplitude baseexcitation.

At low amplitude forcing, the acceleration plots for the three, six, and nine-storey steel buildings are similar to the first-mode response for the MRF. Similarly, for a high amplitude base-excitation, the highest acceleration in the MRF occurs at the maximum height (top of the buildings). Contrarily, in the rocking frames, the acceleration decreases along the building heights, with the maximum magnitudes occurring in the lower half of the buildings. This effect is less defined in the three-storey building and becomes more pronounced for the nine-storey frame. Following this observation, structural response quantities including 

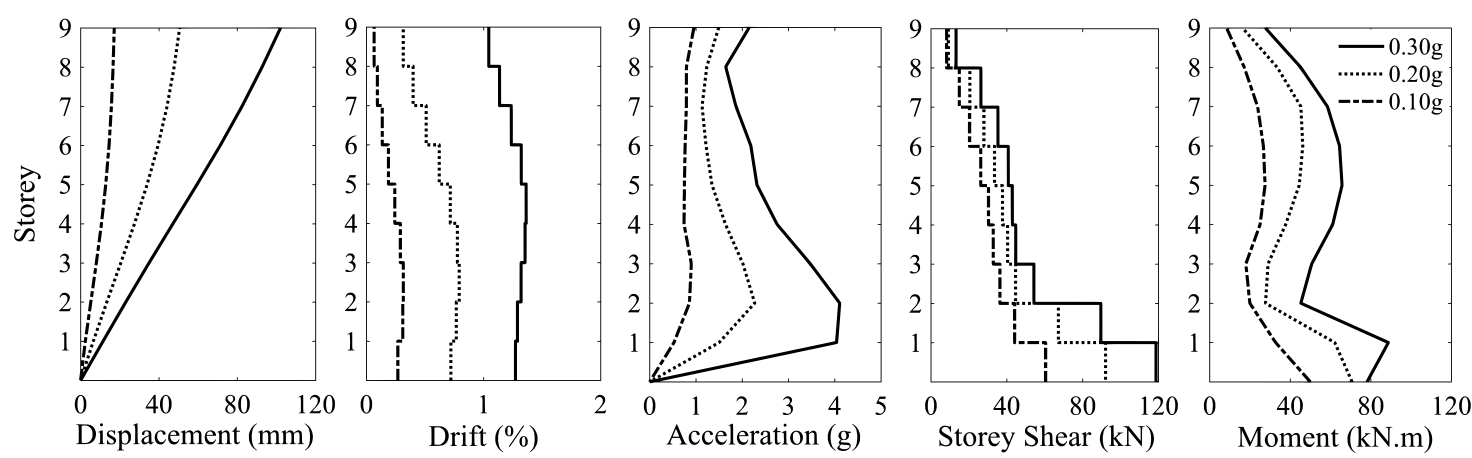

Figure 16: 9-storey structural response quantities for low- $0.10 \mathrm{~g}$, medium- $0.20 \mathrm{~g}$, and high- $0.30 \mathrm{~g}$ harmonic amplitude baseexcitation.
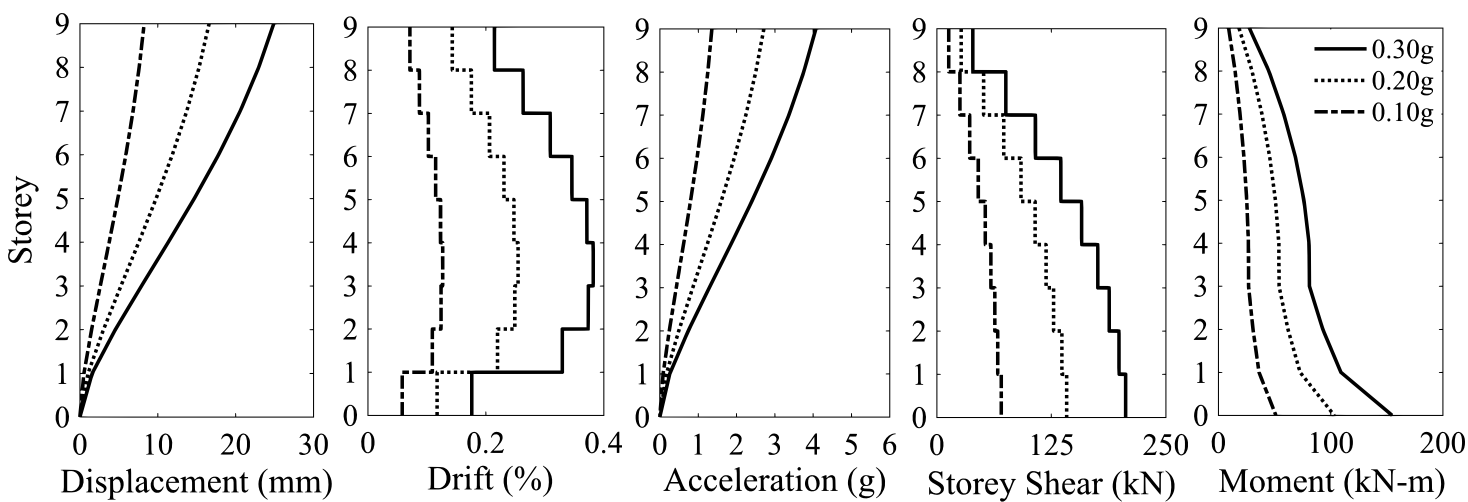

Figure 17: 9-storey fixed-base moment-resisting frame with fully rigid connections for low- $0.10 \mathrm{~g}$, medium- $0.20 \mathrm{~g}-$, and high-0.30g amplitude base-excitation. 

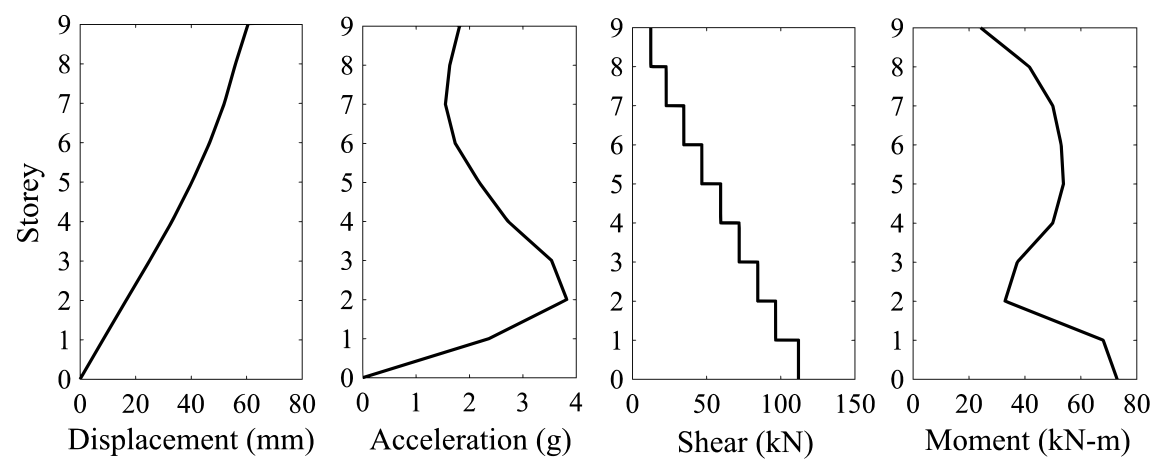

Figure 18: 9-storey structural response quantities corresponding to Point $\mathrm{C}$ for high-0.30g harmonic amplitude base-excitation.
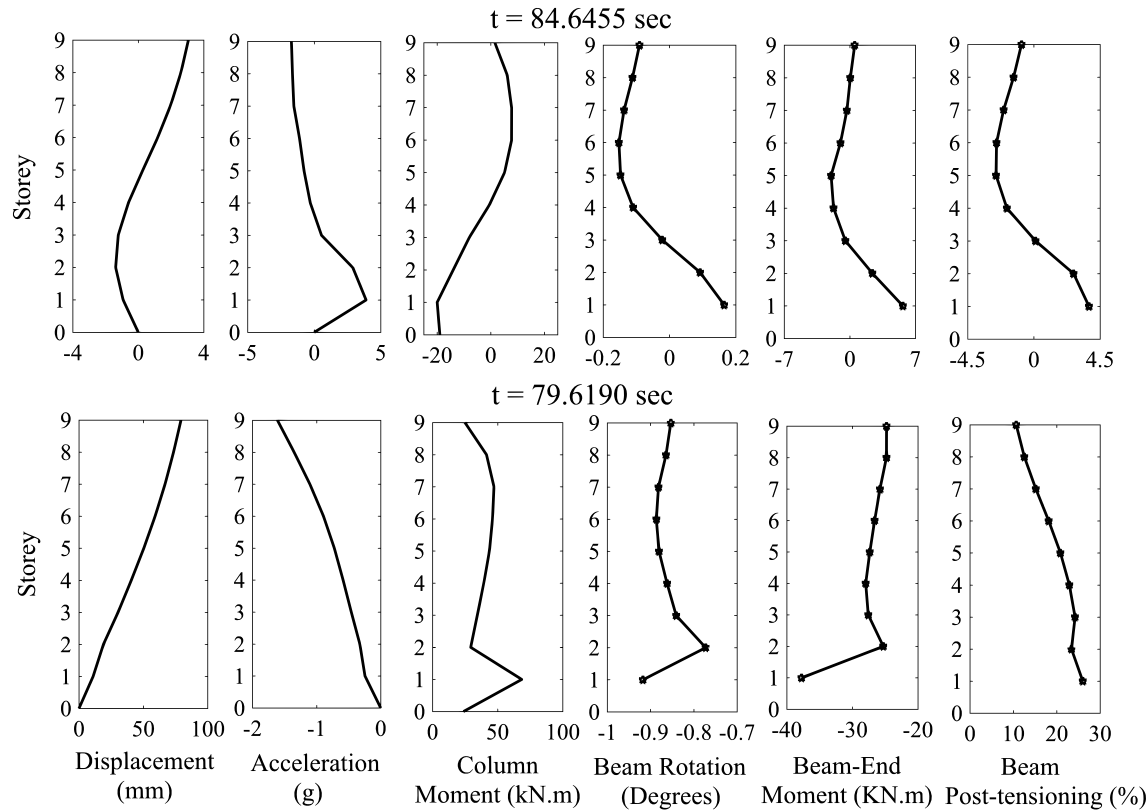

Figure 19: 9-storey response quantities at specific times for high-0.30g amplitude forcing.

the beam rotations, beam-end moments, and beam post-tensioning forces were plotted for specific times in the response history (Figure 19). These quantities are representative of the gap-openings between the beam and column members and could highlight the interactions at these joints. It can be noted from Figure 19 (at $\mathrm{t}=84.65 \mathrm{~s}$ ) that the maximum acceleration at the first storey level occurs when the storey displacements experience a change from a negative to positive slope. At this time, the highest magnitudes for the beam rotations, beam-end moments, column moments, and beam post-tensioning forces are observed at the first storey level, following the maximum acceleration.

The shear forces of the rocking frames are very similar to the conventional MRF response, However, the column bending moments are clearly influenced by effects specific to post-tensioned rocking steel frames. The moment response plots for the rocking frames, presented in Figures 14 to 18 qualitatively indicate interaction effects, particularly around the first and second storeys. A peak in the bending moment envelopes at the resonant frequency and high amplitude forcing, is observed at the first storey for all models with the bending moments stabilising at the second storey. At the first-storey level, the column bending moment magnitude is greater than the value at the base. In comparison, the MRF experiences maximum moments at its base. This peak can be attributed to the interactions between members with different end 
conditions at resonance, when the structure is subjected to high amplitude base-excitation. In the case of the three-storey frame, the bending moment magnitudes at the first-storey govern. It can also be noted that for the nine-storey model, the peak is not observed for Points A and C (Figure 18), which suggests that this characteristic is exclusive to the resonant response of the frame. These beam-column interaction affects can be explained by first examining the response quantities illustrated in Figure 19 (at $\mathrm{t}=79.62 \mathrm{~s}$ ). At this time, the highest beam rotation occurs at the first storey level and decreases along the height of the frame, with a drop in magnitude at the second storey level. The greater beam rotation at the first storey imposes an additional moment on the column thereby leading to higher values. To further explain these beam rotational effects, the frame's member symmetry was examined. Figure 20 illustrates that the first storey column rocks at the base, and is continuous along the remaining structure. The first-storey rocking beam interacts with a rocking column and a continuous column connection, whereas the second storey beam connects into a continuous column at both ends. The discontinuity in the structural connections results in a disturbance in the beam rotations at the first storey. This helps explain the column bending moment peaks at this point in the rocking frames. The multi-storey structures also experience an imbalance of negative and positive moments at the first storey level. Figure 21 illustrates the bending moments at the first-storey column of the nine-storey MRF and rocking frames, subjected to sinusoidal base-motion (Figure 21a). It can be noted that unlike the MRF (Figure 21b), the rocking frame response is not a sinusoidal function. This demonstrates the moment imbalance at these points, and the corresponding structural interactions.

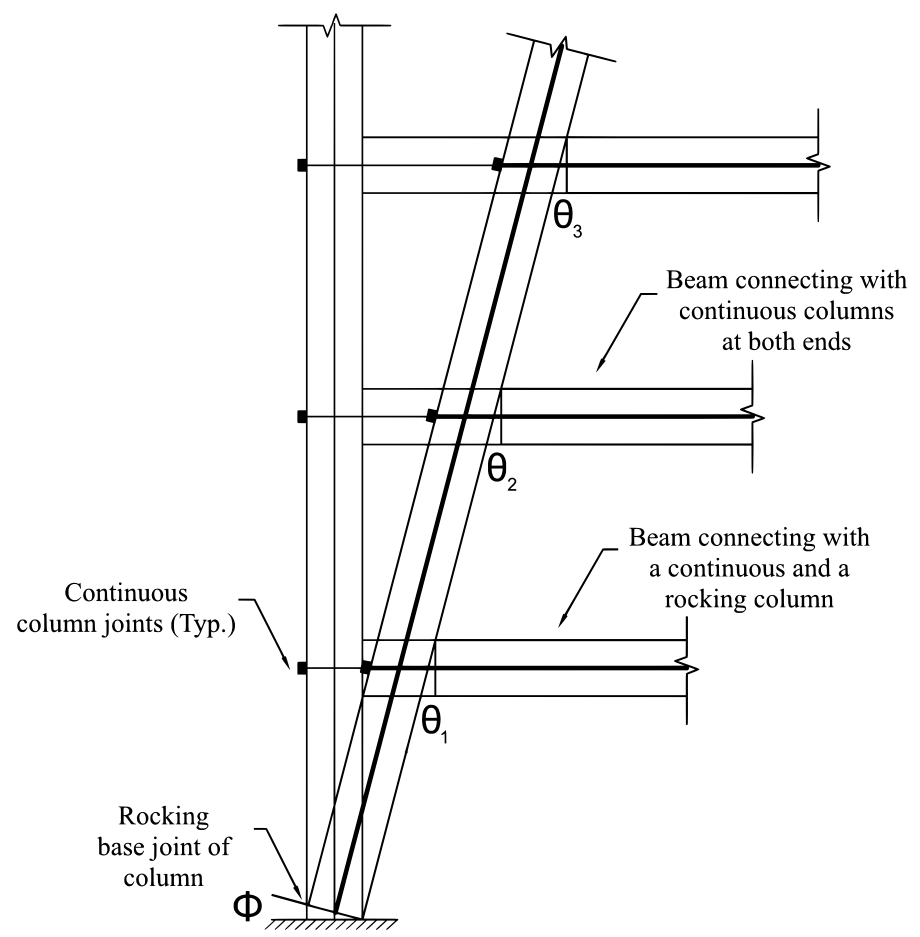

Figure 20: Rocking frame schematic illustrating lateral deformations of the first mode of response

A series of analyses were further conducted altering various design variables in order to understand the bending moment response peaks. It was discovered that when the diameter of the post-tensioning cable was increased by a factor of 1.4, the peak was not observed for any of the buildings. The curves in the acceleration and upper-half of the bending moment plots were still observed but were less pronounced. This indicates that when the lateral stiffness of the structure is increased, the rotation at the base-rocking joint decreases, resulting in lower rotations for the beam-column rocking joints, hence eliminating the peak and verifying the initial hypothesis. 


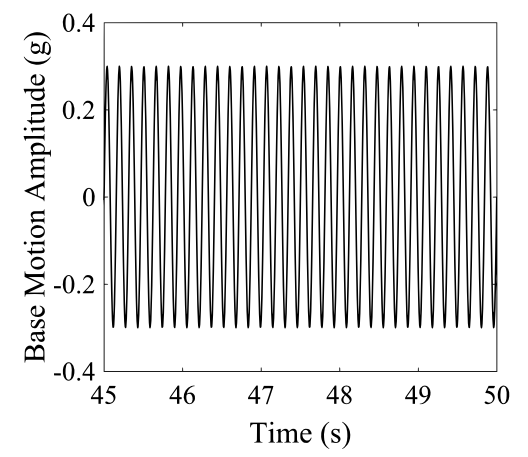

(a) Sinusoidal base-excitation with an amplitude of $0.30 \mathrm{~g}$.

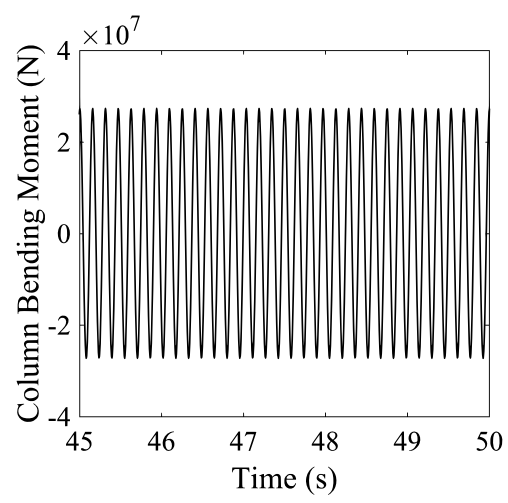

(b) MRF column bending-moments.

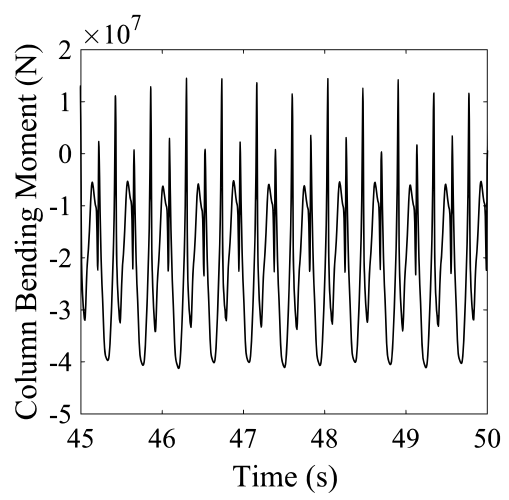

(c) Rocking steel frame column bendingmoments.

Figure 21: Temporal evolution of column moments at the first storey of 9-storey structures.

\section{Conclusions}

Although preceding research has offered extensive insight into the behaviour of different types of rocking steel structures, they have either considered pure rocking behaviour without post-tension self-centring, or have dealt with structures that incorporate considerable levels of supplemental energy-dissipation, which dampens the fundamental dynamic nonlinearity within the rocking response. Consequently, the complex nonlinear dynamic behaviour of single and multi-storey tied rocking steel frames without supplemental damping by means of numerical tools have not been explored previously.

To address these shortcomings, the Finite Element programme, OpenSees, is used in this study to compile and establish a complete numerical modelling strategy (Section 2) for analysing the nonlinear dynamic response of post-tensioned rocking steel moment frames. For this purpose, recommendations are presented for variables affecting the stiffness distribution at rocking interfaces. It is proposed that dividing the length of the rocking surface by a factor of 10 provides a suitable baseline for the number of gap elements along a rocking surface. Moreover, initial estimates for the influence lengths used to calculate individual spring stiffnesses are suggested to be four times the column and twice the beam section depths.

The results of a selected experimental study were numerically simulated in order to validate the Finite Element modelling methodology, and compared with discrete-element and analytical estimations. A comparison of the static and dynamic behaviour obtained confirms that the finite element approximation offers a good representation of the nonlinear dynamics of the rocking steel frame. At both high and low level base excitations, the shape and response amplitudes from the finite-element model are highly similar to the experimental results and analytical approximations. Likewise, the finite element solutions overlap the analytical frequency response functions over a wide frequency range, particularly for the increasing frequency branch. The fundamental frequency of the finite-element model is close to that of the analytical estimation and physical experiments. Altogether, the finite-element model is able to predict the full nonlinear response of the rocking frames more accurately than its discrete-element counterpart.

The modelling procedures were extended to multi-storey rocking steel frames and the resulting behaviour was investigated. Numerical models comprising three, six, and nine-storeys were developed. The static response of the buildings demonstrates that an increase in the initial force and/or area of the column post-tensioning tendons may have a detrimental impact on the structural response of rocking frames. The 
nonlinear dynamic behaviour of the steel frames suggests that the accelerations and bending moment magnitudes are influenced by beam-column interactions effects. These effects are defined to be the consequence of the discontinuity of the member connections and the asymmetry in member restraints due to the presence of base-rocking. The bending moment values due to these effects are pertinent and in many cases, will govern the design of this joint.

Lastly, by comparing the results of the nine-storey rocking steel frame against its moment-resisting frame counterpart with fully-rigid connections, it is demonstrated that the rocking motion leads to relatively larger storey displacements in comparison with the linear-elastic and nonlinear-elastic models of moment-resisting frames. However, rocking also significantly limits the bending moments and shear forces experienced by the primary structural members. These effects, which have not been explored in preceding studies and are not accounted for in current steel design codes, are important as they can further lead to contributions towards the provision of practical design guidelines for post-tensioned rocking steel frames.

\section{References}

[1] D. Kellenberg, A. Mobarak, The Economics of Natural Disasters, Annual Review of Resource Economics 3 (1) (2011) 297-312.

[2] N. Chancellor, M. Eatherton, D. Roke, T. Akbas, Self-Centering Seismic Lateral Force Resisting Systems: High Performance Structures for the City of Tomorrow, Buildings 4 (3) (2014) 520-548.

[3] M. DeJong, E. Dimitrakopoulos, Dynamically equivalent rocking structures, Earthquake Engineering \& Structural Dynamics 43 (10) (2014) 1543-1563.

[4] N. Makris, M. Vassiliou, The Dynamics of the Rocking Frame, Seismic Assessment, behavior and Retrofit of Heritage Buildings and Monuments 37 (4) (2015) 37-60.

[5] M. Vassiliou, K. Mackie, B. Stojadinović, A finite element model for seismic response analysis of deformable rocking frames, Earthquake Engineering \& Structural Dynamics 46 (3) 447-466.

[6] M. Priestley, Overview of PRESSS Research Program, PCI Journal 36 (4) (1991) 50-57.

[7] C. Christopoulos, A. Filiatrault, C.-M. Uang, B. Folz, Posttensioned Energy Dissipating Connections for Moment-Resisting Steel Frames, Journal of Structural Engineering 128 (9) (2002) 1111-1120.

[8] R. Sause, J. Ricles, D. Roke, C.-y. Seo, K.-s. Lee, Design of Self-Centering Steel Concentrically Braced Frames, Proceedings of the 4th International Conference on Earthquake Engineering Taipei, Taiwan.

[9] T. L. Karavasilis, S. Kerawala, E. Hale, Hysteretic model for steel energy dissipation devices and evaluation of a minimaldamage seismic design approach for steel buildings, Journal of Constructional Steel Research 70 (2012) 358 - 367.

[10] M. Newcombe, Seismic Design of Post-Tensioned Timber Frame and Wall Buildings, PhD Thesis Department of Civil and Natural Resources Engineering, University of Canterbury, New Zealand.

[11] L. Wiebe, C. Christopoulos, R. Tremblay, M. Leclerc, Mechanisms to limit higher mode effects in a controlled rocking steel frame. 1: Concept, modelling, and low-amplitude shake table testing, Earthquake Engineering and Structural Dynamics 42 (7) (2013) 1053-1068.

[12] J. Ricles, R. Sause, M. Garlock, C. Zhao, Posttensioned Seismic-Resistant Connections for Steel Frames, Journal of Structural Engineering 127 (2) (2001) 113-121.

[13] M. Garlock, J. Ricles, R. Sause, Experimental Studies on Full-Scale Post-Tensioned Steel Moment Connections, Proceedings of the 13th World Conference on Earthquake Engineering Vancouver, Canada.

[14] A. Iqbal, S. Pampanin, A. Buchanan, Seismic performance of full-scale post-tensioned timber beam-column connections, Journal of Earthquake Engineering 20 (3) (2015) 383-405.

[15] S. Pampanin, M. Priestley, S. Sritharan, Analytical modelling of the seismic behaviour of precast concrete frames designed with ductile connections, Journal of Earthquake Engineering 5 (3) (2001) 329-368.

[16] A. Palermo, S. Pampanin, A. Carr, Efficiency of simplified alternative modelling approaches to predict the seismic response of precast concrete hybrid systems, Keep concrete attractive: Proceedings of Fib Symposium Budapest, Hungary.

[17] D. Roke, R. Sause, J. Ricles, N. Gonner, Design Concepts for Damage-Free Seismic-Resistant Self-Centering Steel Concentrically Braced Frames, ASCE Structures Congress Austin, Texas, USA.

[18] Y.-C. Lin, R. Sause, J. M. Ricles, Seismic Performance of Steel Self-Centering, Moment-Resisting Frame: Hybrid Simulations under Design Basis Earthquake, Journal of Structural Engineering 4 (11) (2013) 1823-1832.

[19] N. Alexander, O. Oddbjornsson, C. Taylor, H. Osinga, D. Kelly, Exploring the dynamics of a class of post-tensioned, moment resisting frames, Journal of Sound and Vibration 330 (15) (2011) 3710-3728.

[20] O. Oddbjornsson, Dynamics of nonlinear elastic moment resisting frames, PhD Thesis School of Civil Aerospace and Mechanical Engineering, University of Bristol, Bristol, UK.

[21] O. Oddbjornsson, N. Alexander, C. Taylor, R. Sigbjornsson, Numerical and experimental exploration of the fundamental nonlinear dynamics of self-centring damage resistant structures under seismic excitation, 15th World Conference on Earthquake Engineering (15WCEE) Lisbon, Portugal.

[22] M. Priestley, S. Sritharan, J. Conley, S. Pampanin, Preliminary Results and Conclusions From the PRESSS Five-Story Precast Concrete Test Building, PCI Journal 44 (6) (1999) 42-67. 
[23] S. Sritharan, M. Priestley, F. Seible, A Five-Story Precast Concrete Test Building for Seismic Conditions - An Overview, Proceedings of the 12th world conference on earthquake engineering Auckland, New Zealand.

[24] H. Spieth, A. Carr, A. Murahidy, D. Arnolds, M. Davies, J. Mander, Modelling of post-tensioned precast reinforced concrete frame structures with rocking beam-column connections, New Zealand Society of Earthquake Engineering 2004 Conference Canterbury, New Zealand.

[25] D. Wang, A. Filiatrault, Numerical and Experimental Studies of Self-Centering Post-Tensioned Steel Frames, Technical Report MCEER-08-0017 University of Buffalo, USA.

[26] P. Clayton, C.-Y. Tsai, J. Berman, L. Lowes, Comparision of web plate numerical models for self-centering steel plate shear walls, Earthquake Engineering \& Structural Dynamics 44 (12) (2015) 2093-2110.

[27] G. Housner, The behavior of inverted pendulum structures during earthquakes, Bulletin of the Seismological Society of America 53 (2) (1963) 403-417.

[28] A. Carr, User Manual for the 2-Dimensional Version, Ruaumoko Manual Volume 1 Ruaumoko 2D.

[29] I. Itasca Consulting Group, UDEC - Universal Distinct Element Code Version 4.0 Minneapolis, Minnesota, USA.

[30] F. McKenna, Object-oriented finite element programming: Frameworks for analysis, algorithms and parallel computing, $\mathrm{PhD}$ Thesis Department of Civil and Environmental Engineering, University of California Berkeley, United States of America.

[31] I. MathWorks, Matlab: The language of technical computing, MATLAB Primer Massachusetts, United States of America.

[32] A. Giouvanidis, E. Dimitrakopoulos, Seismic Performance of Rocking Frames with Flag-Shaped Hysteretic Behavior, Journal of Engineering Mechanics 143 (5) (2016) 1-13. 